\title{
There is no Silicon-based Life in the Solar System
}

\author{
David T. Jacob
}

Received: 21 October 2014 / Accepted: 26 November 2014 / Published online: 20 January 2015

(c) Springer Science+Business Media Dordrecht 2015

\begin{abstract}
In this article, silicon is discussed as a possible building block for life within the solar system. Using NASA's definition of life: 'chemical system capable of Darwinian evolution', a narrow description is obtained and analyzed. The possibilities of silicon life are then explored by describing its bonding characteristics, ability to withstand extreme temperatures, and abundance. Titan is investigated as a possible location where silicon-based life would seem possible. Its extremely cold temperatures and lack of liquid water appear to be ideal for silicon-based life to form. After further investigation though, the presence of carbon and rarity of silicon on Titan lead to the planet not being as ideal as first thought. It is also explained that silicon simply does not have the advantages in bonding, chirality, and universal abundance that carbon has. In the end, silicon is ruled out as a possible basis for life in this solar system.
\end{abstract}

Keywords Silicon-based · Silicon-life · Titan · Life-forms

There has always been a constant search for life beyond the carbon-based life that we currently know on Earth. The idea that there could be life-forms out there in the solar system that are not based upon carbon molecules has intrigued many scientists over the centuries. The possibility of silicon compounds being a building block for life took hold in the 1890's when Frederic Stanley Kipping experimented with the element and James Emerson Reynolds gave his address to the British Association for the Advancement of Science $[1,2]$. The newfound knowledge that silicon had

D. T. Jacob $(\bowtie)$

University of Cincinnati, Cincinnati, OH 45221, USA

e-mail: jacobdt@mail.uc.edu the ability to form into long chains, stabilize at extreme temperatures, and, in some ways, act rather similarly to carbon, gave scientists tremendous hope that silicon could be one of a number of elemental building blocks of life outside of carbon. Although silicon showed initial promise, as scientists began to reveal more information on the surrounding planets and moons, all observations pointed to silicon not being a base for life in the immediate solar system.

Before determining if there are silicon-based life-forms out there in the solar system, there first needs to be a set definition of 'life' for this discussion. The MerriamWebster Dictionary is one resource that contains many ways to define 'life', but there needs to be only one chosen in order to keep the discussion as narrow as possible. Although 'a principle or force that is considered to underlie the distinctive quality of animate beings' could work for some cases, 'an organismic state characterized by capacity for metabolism, growth, reaction to stimuli, and reproduction' is sufficiently narrow to probably be a better definition for what makes something 'alive' rather than 'life' [3]. In 1994, a group from NASA stated their own definition of life, simply as a 'chemical system capable of Darwinian evolution'. This definition takes into account all possibilities of 'life' even beyond what we conceive as 'life' [4].

The reason silicon is often projected as a possible element for the basis of life is because it shares several of the same traits and behaviors as carbon. Silicon sits immediately below carbon in Group 14 on the periodic table [1]. This means that silicon has four valence electrons just like carbon, but it also has a larger density. It can attach itself with oxygen, nitrogen, and carbon creating somewhat lengthy molecular chains. Silicon-silicon chains can have both functionalized and non-functionalized side chains, as does carbon-carbon chains [5]. It has also been discovered that pi-conjugated carbon compounds and 
sigma-conjugated polysilanes have similar electrical characteristics [6]. Silicon is relatively abundant on earth as well with approximately $87 \%$ of the earth's crust being silicon oxide compounds [7]. Silicon compounds can even stand up to intense heat and cold temperatures where carbon compounds would break apart [8]. This makes the idea of silicon-based life seem very likely within the solar system. Although it is plentiful on earth, the chances of it producing life here under the earth's environment and temperatures are not ideal.

There remains one location within the solar system where silicon-based life has looked promising. Titan is the largest moon orbiting Saturn and is extremely cold ( $\sim 94 \mathrm{~K})$. This moon has been thought by scientists to possibly be the best chance for harboring silicon-based life. Titan is radically different from the earth and that would appear to be an improvement when trying to cultivate silicon-based life-forms. Titan does have water but it is frozen solid [6]. This is more hospitable for silicon compounds, other than silicon oxide, which normally separate in the presence of liquids like water due to weak bond strength [5]. There is also no oxygen in the atmosphere, which keeps silicon from reacting instantaneously with any oxygen present in air [6]. Ethane and methane are plentiful all over the surface of Titan and methane just happens to be a good solvent for silicon [6]. If there were to be silicon-based life somewhere in the immediate solar system, it would appear to be on Titan.

Although Titan looks like a more suitable place for silicon to thrive, there are several reasons why silicon-based life would not be possible on Titan. Carbon is a big reason. Carbon is still present on Titan, and carbon still produces stronger bonds than silicon, bond energy of $346 \mathrm{~kJ} / \mathrm{mol}$ compared to $222 \mathrm{~kJ} / \mathrm{mol}$. There is also the fact that silicon is mostly down deep in Titan's core [6]. Regardless of the conditions on Titan, silicon just does not have the advantages that carbon has. Carbon forms very stable bonds with itself, but silicon-silicon bonds are slightly weaker and less stable. Handedness (chirality) is also something that silicon does not do too well. Carbon compounds can have either right or left forms, which make it possible for enzymes to register and process them as is the case with amino acids and proteins. Silicon cannot produce many compounds that exhibit handedness, which is a crucial characteristic of interconnected chains that support life [2]. Silicon molecules are usually achiral, which means they can only exhibit one handedness. Carbon and oxygen pair well together. Carbon dioxide gas is the result when carbon is oxidized. When silicon, which has a powerful attraction to oxygen, is oxidized, it produces a solid. This would be a big barrier to any conceivable idea of respiration [2]. Lastly, silicon is actually pretty rare compared to carbon. The amount of carbon out in space is approximately twenty times greater than that of silicon [9].

Silicon's advantages are strong, and it continues to be a valuable element on the earth and within the solar system. However, the limited areas where silicon life-forming conditions are even believed to be possible do not make up for silicon's inability to be a better building block for life than carbon [10]. It is believed that silicon may have played an important role in the beginning of life on earth, but it failed to be the basis for life. It is unknown whether scientists might one day find silicon-based life in our solar system. What is known is that it will be in an environment extremely different than our own.

Acknowledgments I would like to thank Dr. Stephen John Clarson and his Biomedical Materials (MTEN 6065) class for providing me the inspiration to write this article and for his kind assistance with the editing of it. I would also like to thank my wife, Ashley Jacob, for staying up late at night and proofreading this article and offering her knowledge on the issue.

\section{References}

1. Dessy R (1998) Could silicon be the basis for alien life forms, just as carbon is on earth? Scientific American. Scientific American, Inc. Accessed 13 Oct. 2014

2. Darling D (2014) Silicon-based life. Encyclopedia of science. The Worlds of David Darling

3. Life (2014) Merriam-Webster.com

4. Benner SA, Ricardo A, Carrigan MA (2004) Is there a common chemical model for life in the universe? Curr Opin Chem Biol 8.6:672-689

5. Rampelotto PH (2010) The search for life on other planets: Sulfurbased, silicon-based, ammonia-based life. J Cosmol 5:818-827

6. Schulze-Makuch D (2013) Is silicon-based life possible? Air \& Space Magazine. Smithsonian Institution. Accessed 13 Oct 2014

7. Kondratyev MS, et al. (2013) 18 Possible extraterrestrial life: a quantum-chemical look on the silicon analogs of carbon biomolecules. J Biomol Struct Dyn 31.sup1:10-11

8. Chaisson EJ (2013) Alternative biochemistries. Cosmic evolultion: from big bang to humankind. Harvard University Press, Cambridge, MA. Aceessed 13 Oct 2014

9. Owen T (1980) The search for early forms of life in other planetary systems: future possibilities afforded by spectroscopic techniques Strategies for the search for life in the universe. Springer, Netherlands, pp 177-185

10. Clarson SJ (2009) Charles Darwin and silicon. Silicon 1:59-63. doi:10.1007/s12633-009-9020-4 